ARTICLE

Received 20 Feb 2014 | Accepted 8 Aug 2014 | Published 15 Sep 2014 DOl: 10.1038/ncomms5941

\title{
Sculpting carbon bonds for allotropic transformation through solid-state re-engineering of $-\mathrm{sp}^{2}$ carbon
}

Hyun Young Jung ${ }^{1}$, Paulo T. Araujo ${ }^{2, \dagger}$, Young Lae Kim', Sung Mi Jung ${ }^{2}$, Xiaoting Jia ${ }^{3}$, Sanghyun Hong ${ }^{1}$, Chi Won Ahn ${ }^{4}$, Jing Kong ${ }^{2}$, Mildred S. Dresselhaus ${ }^{2,5}$, Swastik Kar ${ }^{6}$ \& Yung Joon Jung ${ }^{1}$

Carbon forms one of nature's strongest chemical bonds; its allotropes having provided some of the most exciting scientific discoveries in recent times. The possibility of inter-allotropic transformations/hybridization of carbon is hence a topic of immense fundamental and technological interest. Such modifications usually require extreme conditions (high temperature, pressure and/or high-energy irradiations), and are usually not well controlled. Here we demonstrate inter-allotropic transformations/hybridizations of specific types that appear uniformly across large-area carbon networks, using moderate alternating voltage pulses. By controlling the pulse magnitude, small-diameter single-walled carbon nanotubes can be transformed predominantly into larger-diameter single-walled carbon nanotubes, multi-walled carbon nanotubes of different morphologies, multi-layered graphene nanoribbons or structures with $\mathrm{sp}^{3}$ bonds. This re-engineering of carbon bonds evolves via a coalescence-induced reconfiguration of $\mathrm{sp}^{2}$ hybridization, terminates with negligible introduction of defects and demonstrates remarkable reproducibility. This reflects a potential step forward for large-scale engineering of nanocarbon allotropes and their junctions.

\footnotetext{
${ }^{1}$ Department of Mechanical and Industrial Engineering, Northeastern University, Boston, Massachusetts 02115, USA. ${ }^{2}$ Department of Electrical Engineering and Computer Science, Massachusetts Institute of Technology, Cambridge, Massachusetts 02139, USA. ${ }^{3}$ Department of Materials Science and Engineering, Massachusetts Institute of Technology, Cambridge, Massachusetts 02139, USA. ${ }^{4}$ National Nanofab Center, Korea Advanced Institute of Science and Technology, 335 Gwahangno, Daejeon 305-806, South Korea. ${ }^{5}$ Department of Physics, Massachusetts Institute of Technology, Cambridge, Massachusetts 02139, USA. ${ }^{6}$ Department of Physics, Northeastern University, Boston, Massachusetts 02115, USA. † Present address: Department of Physics and Astrophysics, University of Alabama, Tuscaloosa, Alabama 35487, USA. Correspondence and requests for materials should be addressed to M.S.D. (email: millie@mgm.mit.edu) or to S.K. (email: S.Kar@neu.edu) or to Y.J.J. (email: jungy@coe.neu.edu).
} 
C arbon nanotubes (CNTs) have attracted considerable interest over the past two decades as they offer a unique combination of physical properties and chemical stability ${ }^{1}$. Particularly, CNT networks are anticipated to be used in broader applications, such as reinforcements for lightweight and high performance composites, multifunctional membranes, electronics and electrodes for energy storage devices ${ }^{2-6}$. However, most assembled CNT networks are mainly based on weak van der Waals interactions between the CNTs in CNT arrays ${ }^{7}$. As a result, the reported experimental mechanical strength, and electrical and thermal conductivities are several orders of magnitude lower than theoretical predictions due to a lower mechanical pulling resistance between nanotubes, and a higher electron and phonon scattering at these 'unconnected' junctions. Recently, there has been significant success and a growing interest in transforming these van der Waals interactions into covalently bonded molecular junctions ${ }^{8-29}$. For example, electron ${ }^{11,19-21}$ and ion ${ }^{11}$ irradiation as well as electrical current sources ${ }^{23-27}$ have been used to modify the structure and morphology of nanocarbons. However, these rearrangement studies were carried out only for local changes of junctions in a few individual nanotubes ${ }^{1-21,25}$ and by the destruction of carbon layers on electrical breakdown ${ }^{23,26,27}$. Furthermore, the reported reconstruction methods require high to extremely high temperature $\left(750-2,200^{\circ} \mathrm{C}\right)$ conditions ${ }^{20,22,28}$, making them power-intensive, and incompatible with various scalable processes. Therefore, developing a fast and scalable method for reproducibly creating particular types of covalently bonded C-C junctions and $\mathrm{sp}^{2}$ molecular structures in nanocarbon networks that result in repeatable physical properties is still a fundamental challenge.

Here we report a method for constructing $\mathrm{sp}^{2}$ inter-nanotube junctions and desired allotropic structures by applying controlled alternating voltage pulses across single-walled carbon nanotube (SWCNT) networks. Transformations across entire networks (electrode to electrode) were characterized point-by-point with Raman spectroscopy and were also visually inspected using transmission electron microscopy (TEM) before and after the voltage pulse cycling was completed. Our transformation process has several interesting features. First, by controlling the electrical parameters, such as the amplitude, the number of cycles and the duty cycle of an alternating voltage pulse, nearly the entire Raman and TEM-inspected regions of SWCNT networks were found to be transformed uniformly and reproducibly, mostly into either larger diameter SWCNTs, multi-walled carbon nanotubes (MWCNTs) or multi-layered graphene nanoribbons (MGNRs). Second, the new nanocarbon network retains its $\mathrm{sp}^{2}$ covalent bonds and seamless structures, without increasing structural defects after the transformational process is completed. Third, the method is facile, easily scalable and extendible into 2-3 dimensional ${ }^{29}$ and multiscale (nano- to macro-scale) applications.

\section{Results}

$I-V$ characterization and alternating voltage pulses. Highly organized SWCNT micronetworks containing about $(2 \pm 0.5)$ $\times 10^{4}$ CNTs per $\mu \mathrm{m}^{2}$ were assembled by using a template-guided fluidic assembly process ${ }^{30-32}$ and attached with electrodes for electrical treatment. Particularly for TEM observation, welldefined SWCNT microlines (see Method section) were directly assembled on specially designed TEM grids where microwindows were located between built-in micro-heaters for external heating. Figure la shows the scanning electron microscopy (SEM) image of an assembled SWCNT network microline between two electrodes. Initial $I-V$ characterization was performed to find the maximum current density $\left(J_{\mathrm{b}}\right)$ and the breakdown voltage $\left(V_{\mathrm{b}}\right)$ of assembled SWCNT networks (Fig. 1b) under vacuum. Typically, the CNT arrays assembled on the TEM windows (Supplementary Fig. 1a,b) failed at current densities of a few tens of $10^{6} \mathrm{~A} \mathrm{~cm}^{-2}$ corresponding to values of $2.5 \mathrm{~V}<V_{\mathrm{b}}<3 \mathrm{~V}$. Figure 1c schematically represents the time-dependent alternating voltage pulses applied on the SWCNT devices. To initiate molecular junction formation and
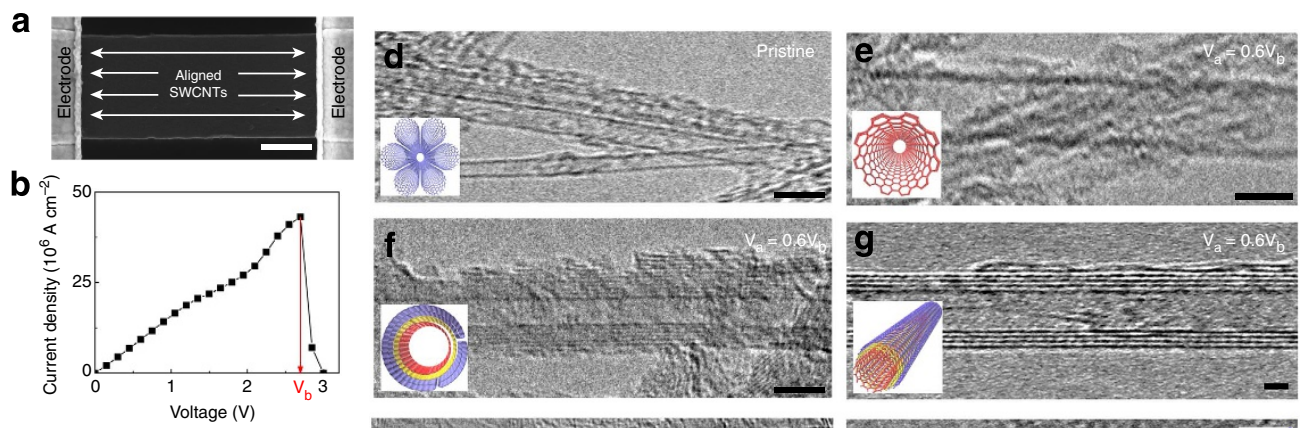

c
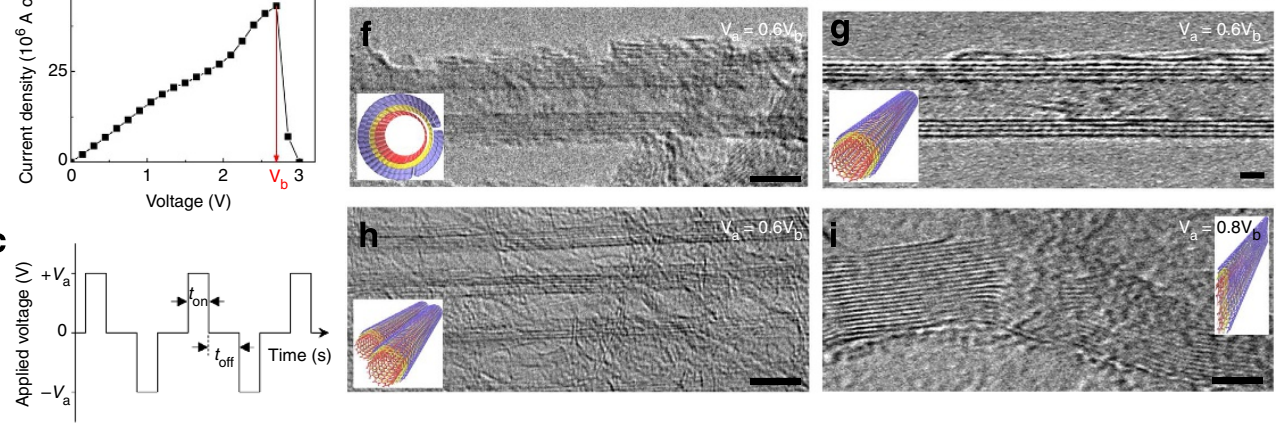

Figure 1 | Pulsed-alternating-voltage induced allotropic transformation of SWCNTs. (a) SEM image of an assembled SWCNT network integrated with electrodes fabricated on a specially designed TEM grid (see Supplementary Fig. 1a). A scale bar is $500 \mathrm{~nm}$. (b) Typical I-V characteristics of a pristine SWCNT network device showing breakdown behaviour beyond the voltage, $V_{b}=2.7 \mathrm{~V}$, corresponding to a maximum current density of about $4.3 \times 10^{7} \mathrm{~A} \mathrm{~cm}^{-2}$. (c) Time-dependent alternating voltage pulses that were used to obtain the described transformation of the SWCNT arrays. $V_{\mathrm{a}}$, $t_{\text {on }}$ and $t_{\text {off }}$ indicate applied voltage amplitude, and voltage ON and OFF durations, respectively. (d) Typical TEM image of the SWCNTs in the array before the voltage cycling was initiated. (e-i) Typical morphologies of nanocarbon structures obtained after the voltage pulse cycling was completed. In each case, $V_{a}$ is presented in units $V_{b}$. The typical carbon morphologies obtained after cycling 3,000 times at $V_{a}=0.6 V_{b}$ are $(\mathbf{e}) S W C N T s$ with a larger diameter, (f) multi-walled tubular structures with several incomplete $\mathrm{sp}^{2}$ walls, ( $(\mathbf{g})$ well-formed MWCNTs and (h) bundles of MWCNTs. (i) MGNRs were obtained typically after the network was subjected to 3,000 cycles of $V_{a}=0.8 V_{b}$. Scale bars in $(\mathbf{d}-\mathbf{i})$ are all $5 \mathrm{~nm}$. 
structural evolution in the SWCNT networks, the electrical polarity was switched periodically (typically at $1 \mathrm{~Hz}$ for 3,000 cycles) for several applied voltage amplitudes $\left(V_{\mathrm{a}}\right.$, Fig. 1c) ranging from $0.4 V_{\mathrm{b}}$ to $0.8 V_{\mathrm{b}}$, where the $I-V$ curves turn slightly nonlinear (Fig. 1b) because of increased electron-phonon scattering and structural changes ${ }^{33}$. The source-on-time $\left(t_{\text {on }}\right.$ in Fig. 1c) for each $V_{\mathrm{a}}$ was also varied to obtain the most effective transformation conditions in SWCNT networks, as discussed later.

Allotropic transformations of carbon nanostructures. Figure 1d shows a typical TEM image of the pristine SWCNT network (that is, before electrical treatment) comprising of individual and bundles of SWCNTs. Figure 1e-i shows TEM images outlining representative results after the cycling steps were completed using different values of $V_{\mathrm{a}}$. Two different regimes were observed as the $V_{\mathrm{a}}$ is increased; one is from 0 to $0.4 V_{\mathrm{b}}$, where $I$ versus $V$ is linear and in which no significant structural changes occur. The other regime is from $0.4 V_{\mathrm{b}}$ to $0.8 V_{\mathrm{b}}$, where interesting structural/ morphological changes in the nanotube array take place along with active rearrangement of carbon atoms. Three different $\mathrm{sp}^{2}$ carbon nanostructures are predominantly observed at the applied voltages of $0.6 V_{\mathrm{b}}$ and $0.8 V_{\mathrm{b}}$. At $V_{\mathrm{a}}=0.6 V_{\mathrm{b}}$, we find that a few of the SWCNT bundles coalesce to form few larger diameter SWCNTs, as seen in Fig. 1e. However, under the same conditions, most of the SWCNT networks are transformed either to MWCNT arrays ranging from 15 to $30 \mathrm{~nm}$ in diameters with several incomplete $\mathrm{sp}^{2}$ outer tube walls (Fig. 1f; which give rise to either dangling bonds and structural imperfections that may enhance the coalescence of CNTs $)^{14,20,25,34-37}$ or to well-defined MWCNTs with hollow cores and completely formed tube-wall (as straight lattice fringes in Fig. 1g,h). For $V_{\mathrm{a}}=0.8 V_{\mathrm{b}}$, multilayered and flattened graphitic structures were most commonly observed (Fig. 1i), which most probably results when largerdiameters single- or few-walled nanotubes gain stability by collapsing into MGNRs. These results represent the typical observations from over 500 different devices tested for scientific exactitude (see more examples in Supplementary Fig. 2).

The large-scale uniform transformation across the entire SWCNT networks implies that during the transformation process, the organized arrangement of individual nanotubes was similar across the entire network. This is partially because individual nanotubes were aligned into narrow (approximately few nanometre) bundles because of coherent capillary forces they undergo during the fluidic assembly process ${ }^{30}$. Further, during the initial cycles of voltage-pulsing, randomly oriented SWCNT bundles were observed to form larger (approximately tens of nanometres) and more stable and aligned bundle configurations. A narrow adjacent distance between nanotubes often promotes the hexagonal lattice packing of SWCNTs into larger bundles and to eventually form a bundled rope. Each pair of nanotubes in this arrangement has almost the same probability of forming a coalescence transformation as the voltage is increased. The dense mechanical alignment of SWCNTs in the initial phase of voltage-pulsing placed each of the nanotubes in intimate contact with its neighbour-a necessary prerequisite for efficient coalescence of the nanotubes. We speculate that most of the energy given to the system for values of $V_{\mathrm{a}}$ up to $0.4 V_{\mathrm{b}}$ is used in this 'stability search' towards better alignment of the nanotubes with each other and to the enlargement of SWCNT bundles without changing their structure (see Supplementary Fig. 2b). Beyond this voltage $\left(V_{\mathrm{a}}>0.4 \mathrm{~V}\right)$, the original $\mathrm{sp}^{2}$ bonds undergo a massive rearrangement, accompanied by the rise of a strong coalescence-induced modes (CIMs) in the Raman spectra, which is a signature precursor for inter-tube coalescence $^{13,38-40}$, details of which is discussed next.
Raman spectra of transformed carbon structures. To further investigate the $\mathrm{sp}^{2}$ junction formation and molecular structure evolution in SWCNT networks, resonant Raman spectroscopy measurements were performed at the same location in the SWCNT network before and after subjecting the sample to the various voltages from $0.2 V_{\mathrm{b}}$ to $0.8 V_{\mathrm{b}}$ (Supplementary Fig. 3). Shown in Fig. 2a are overall Raman spectra showing four representative Raman features: the radial breathing mode (Supplementary Fig. 7), the D-band, the G-band and the $\mathrm{G}^{\prime}$-band, each representing unique spectral evolutions (not previously observed) as the applied voltage is increased. In the same figure, the expected location of a fifth and extremely interesting emergent feature, the $\mathrm{CIM}^{13,38-40}$, has been demarcated using a dashed rectangle. The CIM is a unique identifier of vibrations of linear carbon chains ' $\mathrm{H}-\mathrm{C}_{n}-\mathrm{H}$ ', and depending on ' $n$ ', the length of the chain is expected to result in Raman modes around $\omega_{\mathrm{CIM}}=1,850-1,900 \mathrm{~cm}^{-1}$. The CIMs provide a very clear confirmation that carbon atoms in the nanotube lattice are moving/migrating to form different $\mathrm{sp}^{2}$ carbon structures that are mediated via the temporary appearance of junctions in the form of these linear C-chains between adjacent nanotubes ${ }^{13,38-40}$ as shown schematically in the inset of Fig. 2a. These modes have been determined to be a crucial precursor to the coalescence process in high-temperature-induced coalescence of nanotubes ${ }^{38}$. Interestingly, for our voltage-pulse-induced coalescence, the CIM modes are more prevalent when the pulse duty-cycle $\left(t_{\text {on }} /\right.$ $\left.\left(t_{\mathrm{on}}+t_{\mathrm{off}}\right)\right)$ is smaller. This fact is illustrated in Fig. $2 \mathrm{~b}$, which shows typical Raman spectra near the CIM mode for samples that have been cycled using different 'on times', $t_{\text {on }}$ (keeping $t_{\text {off }}$ constant at $400 \mathrm{~ms}$ ). As $t_{\text {on }}$ decreases, CIM modes (demarcated by arrows in Fig. 2b) begin to appear at $t_{\mathrm{on}}=500 \mathrm{~ms}$, being most prominent at $t_{\mathrm{on}}=100 \mathrm{~ms}$. The disappearance of CIM modes for larger $t_{\text {on }}$ values seem to imply that rapid switching of the voltagepulse is a more effective method to obtain the transformations. Selecting $t_{\text {on }}=100 \mathrm{~ms}$, we have systematically studied the evolution of CIM peak in a large number of samples subjected to different $V_{\mathrm{a}}$ at various cycling stages. As the voltage cycling proceeds, the CIM modes were found to appear and often disappear (see Supplementary Figs 4 and 5) in most samples, which is consistent with the fact that the linear carbon chains are intermediate steps during the transformation of SWCNTs into more complex structures. At $V_{\mathrm{a}}=0.8 V_{\mathrm{b}}$, the CIM peaks were found to be present even after 3,000 cycles implying that at this high voltage, the transformations were much more large-scale, and within our experimental cycling-range, partially complete. This very typical observation has been illustrated in Fig. 2c, which shows that at the end of the 3,000 cycles, a prominent CIM remains around $\omega_{\mathrm{CIM}}=1,855 \mathrm{~cm}^{-1}$ for samples cycled at $V_{\mathrm{a}}=0.8 V_{\mathrm{b}}$. The appearance of the CIM mode is well correlated with our detailed TEM-based inspection of the samples that have been cycled at various values of $V_{\mathrm{a}}$. For samples cycled at $0.4 V_{\mathrm{b}}$ (Supplementary Fig. 4a) and below, where the CIM never shows up, we observe no major structural changes in TEM images. When cycled at $0.6 V_{\mathrm{b}}$, which results in various MWCNT morphologies (Fig. 1e-h), the CIM spectral feature appears intermittently at different cycling stages, for example, at 600 and 2,500 cycles for the sample shown in Supplementary Fig. 4b. However, at $0.8 V_{b}$, where a highly active structural transformation occurs, the CIM appears consistently throughout the entire cycling stage, as shown in Fig. 2d (see more in Supplementary Fig. 5). A clear trend of the rate of structural transformations emerges when the CIM peak intensity $I_{\mathrm{CIM}}$ is plotted as a function of cycles, as seen in Fig. 2e. $I_{\text {CIM }}$ grows steadily with increased voltage cycling, and exhibits a clear peak, at $\sim 1,500$ cycles beyond which the rate of structural transformations gradually slows down, indicating that the 


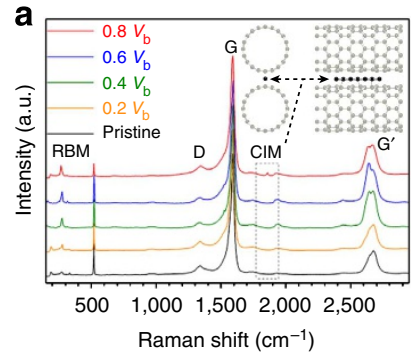

d

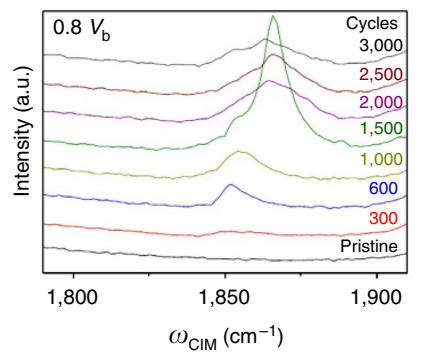

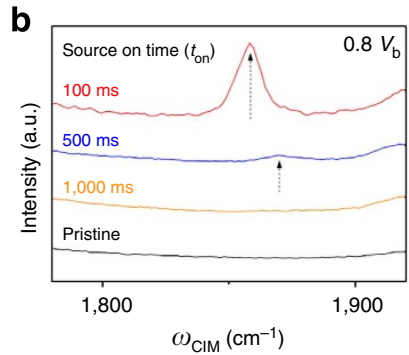

$\mathbf{e}$

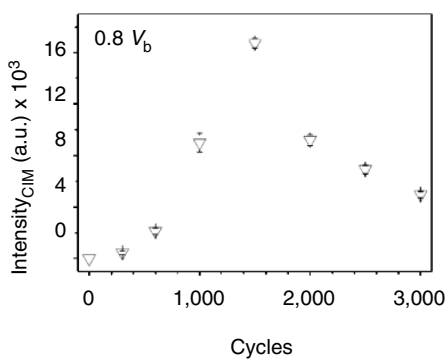

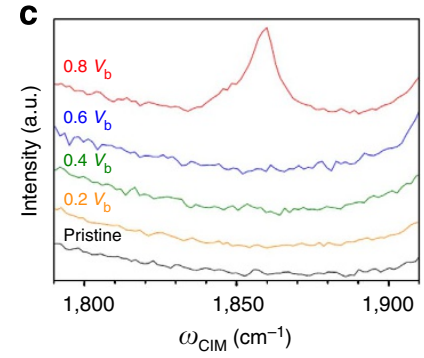

$\mathbf{f}$

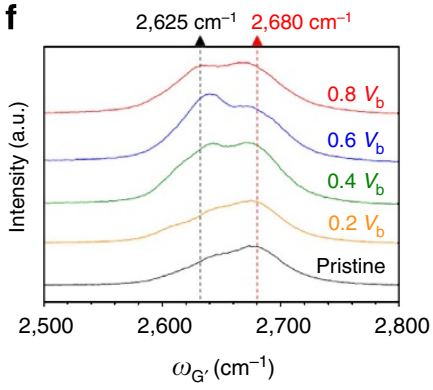

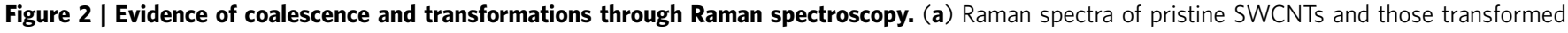
by 3,000 cycles of different pulse-amplitudes $V_{a}$, as noted. The signature peaks (radial breathing mode, G, D and $G^{\prime}$ ) of SWCNTs are labelled. At low duty cycles (that is, smaller values of $t_{\text {on }} /\left(t_{\text {on }}+t_{\text {off }}\right)$ ), a coalescence-induced mode (CIM, demarcated by arrows) was found to emerge in the Raman spectra, as seen here and more prominently in $\mathbf{b}$ for a sample cycled at $V_{\mathrm{a}}=0.8 \mathrm{~V}_{\mathrm{b}}$. $t_{\mathrm{off}}=400 \mathrm{~ms}$ in all cases. (c) After 3,000 cycles, the CIM was found most consistently in samples cycled at $V_{a}=0.8 V_{b}$, as shown. (d) Evolution of the CIM peak as a function of number of cycles. (e) Variation of the CIM peak intensity as a function of number of cycles. The peak maximizes around 1,500 cycles indicating maximum SWCNT transformations occurring mid-way of the total 3,000 cycles. Error bars represent the s.d. (f) Evolution of the $G^{\prime}$ band for samples cycled at various values of $V_{a}$. A low-frequency peak at $\omega=2,625 \mathrm{~cm}^{-1}$ emerges after voltage cycling of SWCNTs, consistent with the appearance of large-diameter single- or multi-walled nanotubes. All spectra were measured with a $532 \mathrm{~nm}$ laser line $(2.33 \mathrm{eV})$.

structural transitions were largely complete. Hence, this cycle dependence along with the $t_{\text {on }}$ dependence enables us to identify the most effective electrical conditions for transforming SWCNT networks into the various $\mathrm{sp}^{2}$ nanostructure SWCNT networks that occur from $0.5 V_{\mathrm{b}}$ to $0.8 V_{\mathrm{b}}$ voltages. In addition to the CIM, we have also investigated the evolution of the $\mathrm{G}^{\prime}$-band of the nanotubes before and after voltage cycling. Figure $2 \mathrm{f}$ shows the line-shape and intensity change in the $\mathrm{G}^{\prime}$-band spectra after electrical treatment with different applied voltages. The average peak position of the $G^{\prime}$-band $\left(\bar{\omega}_{G^{\prime}}\right)$ is known to red-shift to lower frequencies with increasing mean nanotube diameter $\overline{d_{\mathrm{t}}}$ as, $\bar{\omega}_{G^{\prime}}\left(\overline{d_{\mathrm{t}}}\right)=\omega_{\text {graphite }}+C / \overline{d_{\mathrm{t}}}$ (ref. 41). In addition to the pristine $\mathrm{G}^{\prime}$ peak with a maximum around $2,680 \mathrm{~cm}^{-1}$, we find the clear emergence of a red-shifted peak centred around $2,625 \mathrm{~cm}^{-1}$ after voltage cycling, consistent with the coalescence of small diameter SWCNTs, which forms bigger diameter SWNTs/MWNTs, and the collapse of MWCNTs into their layer-stacked MGNR counterparts.

Electrical and thermal properties. In-situ two-terminal electrical resistance changes in SWCNT devices were also measured during the entire transformational process, as such data are indicative of successful junction formation and structural evolution. Our analyses (see Supplementary Fig. 8) show that contact resistances play only a small role in the overall changes. Figure 3 a shows the resistance change as a function of the number of cycles at alternating voltage-pulses of $0.4 V_{\mathrm{b}}, 0.6 V_{\mathrm{b}}$ and $0.8 V_{\mathrm{b}}$, respectively. For samples cycled at $V_{\mathrm{a}}=0.4 V_{\mathrm{b}}$, the electrical resistance is seen to increase slowly over time over the entire cycling period. Since neither TEM nor Raman investigations indicated any structural transformations at this $V_{\mathrm{a}}$ value, and analysis of the D-band of Raman spectrum (Fig. 3b) showed a decrease in defect density, we conjecture that at this voltage, the nanotubes may have been undergoing small mechanical alignment changes that altered the conductive pathways. With increasing $V_{\mathrm{a}}$, however, there was a complete trend-reversal, with the overall resistance decreasing with increasing voltage cycles, commensurate with the increasing inter-nanotube junction formation, as well as significant increase in diameter and wall-thickness of the conductive structures. The decreasing electrical resistance because of voltage cycling is also highly consistent with the steady decrease of defect density in the structures after voltage cycling was completed. This fact is brought out in Fig. $3 \mathrm{~b}$, which shows the variation of $I A_{\mathrm{D}} / I A_{\mathrm{G}}$ (ratio of the integrated areas of the $\mathrm{D}$ and $\mathrm{G}$ bands) as a function of $V_{\mathrm{b}}$. The results clearly show a sizeable decrease in $I A_{\mathrm{D}} / I A_{\mathrm{G}}$ with increasing $V_{\mathrm{a}}$, indicating that the network is getting relatively less defective (relative decrease of D-band intensity) and more organized by forming more ordered $\mathrm{sp}^{2}$ carbon nanostructures (relative increase of G-band) after the transformational process.

Figure $3 \mathrm{c}$ shows the percentage drop in post-cycling resistance as a function of $t_{\text {on }}$ in a sample cycled 3,000 times with $V_{\mathrm{a}}=0.8$ $\mathrm{V}$. Interestingly, the electrical resistance drop was found to be largest when the duty-cycle was smallest, which is in direct agreement with the $t_{\text {on }}$-dependent appearance of the CIMs (Fig. 2b), suggesting that rapid pulse-switching (when $t_{\text {on }}$ is small) rather than prolonged Joule-heating (when $t_{\text {on }}$ is large) better assists in the transformation processes. Figure $3 \mathrm{~d}$ shows the variation of the electrical resistance and thermal conductivity in SWCNT networks as a function of $V_{\mathrm{a}}$ (contact effects were removed using methods described in Method section). Particularly, when $V_{\mathrm{a}}=0.8 V_{\mathrm{b}}$ is applied for 3,000 cycles, both the electrical contact resistance (metal electrodes-SWCNTs)and intrinsic network resistance (see Supplementary Fig. 8) decrease because of the formation of electrically stable metal-nanotube 
a

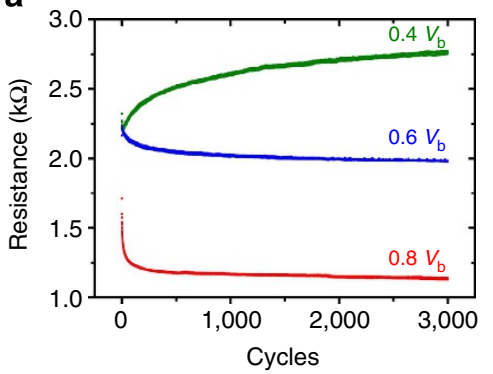

C

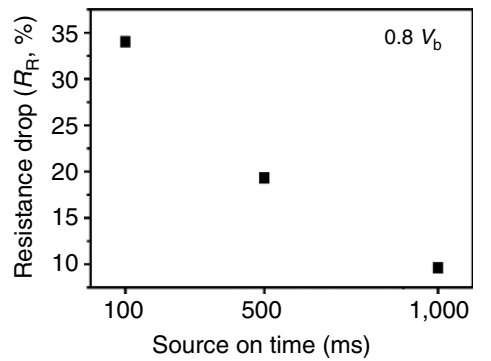

b

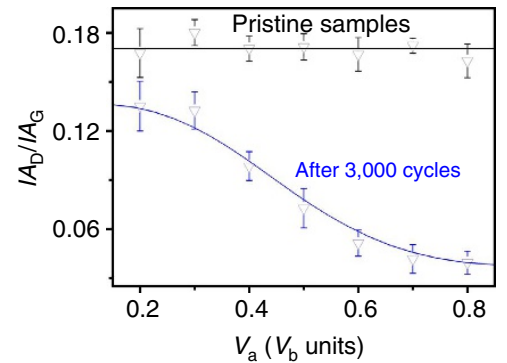

d

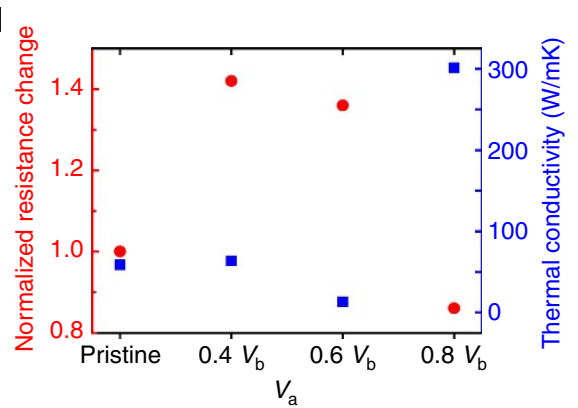

Figure 3 | The electrical and thermal properties. (a) In-situ two-terminal electrical resistance of the SWCNT devices as a function of voltage-pulse-cycling at different $V_{a}$ values, as noted. (b) The ratio of the integrated area under the $D$ and $G$ band $\left(I A_{D} / I A_{G}\right)$ for a range of applied $V_{a}$ values, obtained from Raman spectroscopy before and at the end of 3,000 cycles. This ratio is a measure of disorder density in SWCNTs, which is found to systematically decrease as $V_{\mathrm{a}}$ is increased. Error bars represent the s.d. (c) Percentage device resistance drop, $R_{\mathrm{R}}=\left(R_{\text {before }}-R_{\text {after }}\right) \times 100 / R_{\text {before }}$ after voltage-pulsecycling is applied at $V_{a}=0.8 V_{b}$, for various $t_{\text {on }}$ values. Interestingly, a larger decrease of device resistance was found when the duty-cycle (that is, $t_{\text {on }}$ ) is the smallest, consistent with the data in Fig. 2b. (d) Variation in normalized resistance change $R$ (after subtracting the contact resistance, see Supplementary Fig. 8) and thermal conductivity (calculated based on equation (5) and Supplementary Fig. 9).

interfaces and restructured $\mathrm{sp}^{2}$ nanocarbon networks. At the same time, the post-cycling thermal conductivity was found to gradually decrease from $\sim 60 \mathrm{~W} \mathrm{mK}^{-1}$ to $\sim 10 \mathrm{~W} \mathrm{mK}^{-1}$ up to $V_{\mathrm{a}}=0.6 V_{\mathrm{b}}$. For $V_{\mathrm{a}}=0.8 V_{\mathrm{b}}$, however, there was a dramatic increase in thermal conductivity, reaching values of $\sim 300 \mathrm{~W} \mathrm{mK}^{-1}$, which is five times higher than that of pristine SWCNT networks. The sharp drop in the network electrical resistance and dramatic increase in its thermal conductivity when voltage-pulse cycled at $V_{\mathrm{a}}=0.8 V_{\mathrm{b}}$ is accompanied by a rather unexpected finding-the possible appearance of $\mathrm{sp}^{3}$-hybridized carbon in the network.

$\mathbf{s p}^{2}-\mathbf{s} \mathbf{p}^{3}$ hybrid nanostructures. Figure $4 \mathrm{a}$ shows an SEM image of the network structure after being cycled at $0.8 \mathrm{~V}_{\mathrm{b}}$. High-resolution TEM image of the central portion of this structure (Fig. 4b) reveals that the transformed network consists of a multi-layered graphitic $\mathrm{sp}^{2}$ structure. The measured in-plane lattice spacing in the centre is $0.24 \pm 0.02 \mathrm{~nm}$, which is identical to the $\mathrm{sp}^{2}$ carbon structure $^{24}$. This observation is consistent with all our previous findings. However, carbon K-edge energy loss spectrum (EELS) and resonant Raman spectroscopy results that compare the central region versus the edges of these networks reveal that at the edges the network is transformed to $\mathrm{sp}^{2}-\mathrm{sp}^{3}$ hybrid nanostructures. In Fig. 4c, we compare the EELS spectra from the centre (red) and the edge (blue) regions of the sample shown in Fig. 4a. In general, $\mathrm{sp}^{2}$ bonds exhibit a peak around $290 \mathrm{eV}$ because of $\sigma^{\star}$ states and the other peak appears at $285 \mathrm{eV}$ corresponding to $\pi^{\star}$ states $^{42-44}$, as seen in the spectrum measured from the centre of the sample. However, the EELS spectrum measured at the edges shows clear signatures of $\mathrm{sp}^{3}$-type carbon: a $\sigma^{\star}$ edge around $290 \mathrm{eV}$ corresponding to the $\mathrm{sp}^{3}$ transition $1 \mathrm{~s} \rightarrow 2 \sigma^{*}$ (ref. 45) and a dip around $302 \mathrm{eV}$ that corresponds to the second absolute band gap characteristic of the crystalline $\mathrm{sp}^{3}$ structure ${ }^{46,47}$. Further, the $\mathrm{sp}^{2}$ carbon band at $\sim 285 \mathrm{eV}$ becomes a very shallow shoulder in the spectrum of the $\mathrm{sp}^{3}$-bonded carbon. We have used the relative G-band shift in Raman spectra focused on two different locations, centre and edges of the transformed network (Fig. 4d), to estimate the percentage abundance of $-\mathrm{sp}^{3}$ carbon. The Raman spectra, taken at the centre of networks, zoomed into the G-band range (inset in Fig. 4d) indicate that this transformed network keeps mostly crystalline $\mathrm{sp}^{2}$ bonds. However, at the edges, Raman spectrum shows the characteristics of a nano-crystallization of the pristine material with highly disordered $\mathrm{sp}^{2}$ bonds (amorphization) and the rise of some $\mathrm{sp}^{3}$ bonds. The contents of $\mathrm{sp}^{3}$ bonds (in \%) at the edges can be calculated with the relation ${ }^{48} \mathrm{sp}^{3}(\%)=0.24-$ $0.0049^{\star}\left(\omega_{\mathrm{G}(\text { edge })}-\omega_{\mathrm{G}(\text { centre })}\right)$, in which $\omega_{\mathrm{G}(\text { edge })}=1,602.4 \mathrm{~cm}^{-1}$ and $\omega_{\mathrm{G} \text { (centre) }}=1,590 \mathrm{~cm}^{-1}$ stand for the G-mode Raman frequency at the edges and in the centre of the resultant material. According to this expression, there are about $17 \%$ of $\mathrm{sp}^{3}$ bonds at the edges (as annotated in Fig. 4a). As shown in the Fig. 4a, nanotubes in both edges are more highly aligned because of the capillary force-induced assembly of SWCNTs ${ }^{30,32}$. Highly excited electrons for $V_{\mathrm{a}}=0.8 V_{\mathrm{b}}$ constantly transfer their energy to the nanotube lattice. Especially for the highly aligned SWCNT arrays at the edges of the network, a more effective rearrangement of carbon atoms can potentially occur along the compact and aligned nanotube lattices, leading to the possible formation of the $\mathrm{sp}^{3}$ structures. Therefore, it is assumed that reduced junction scattering and increased thermal conduction pathway through the $\mathrm{sp}^{3}$ carbon structure might cause the previously discussed high thermal conductivity in this network.

\section{Discussion}

The exact mechanism at the atomic-scale that initiates the $\mathrm{C}-\mathrm{C}$ bond reconfigurations is not yet clear to us at this point. 
a

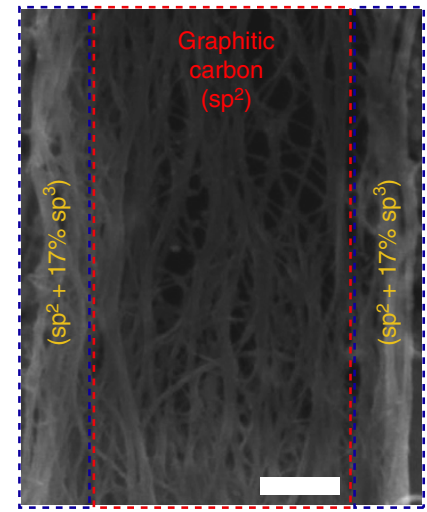

C

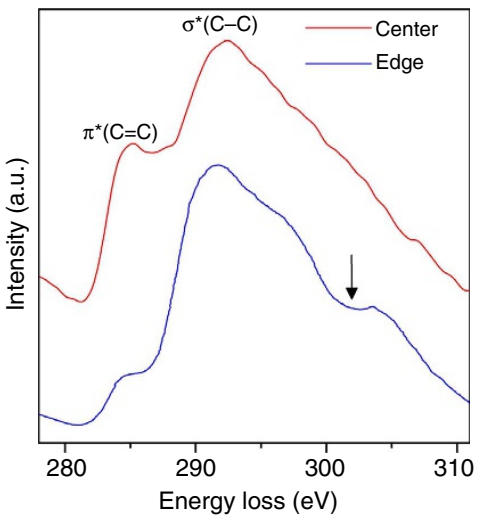

b

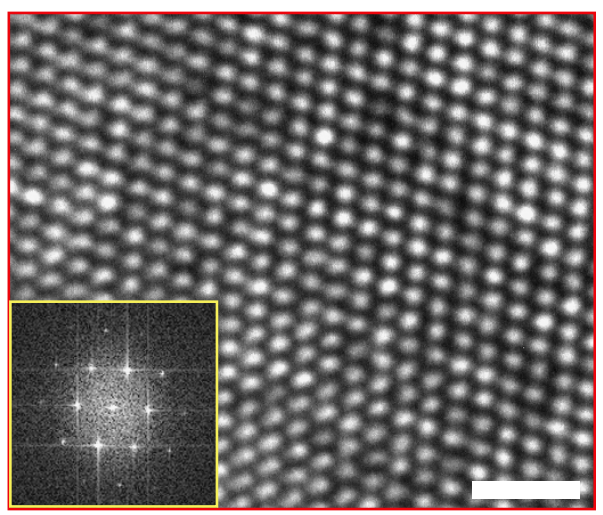

d $\omega_{\mathrm{G}(\mathrm{edge})}-\omega_{\mathrm{G}(\mathrm{bulk})}=12.4 \mathrm{~cm}^{-1}$

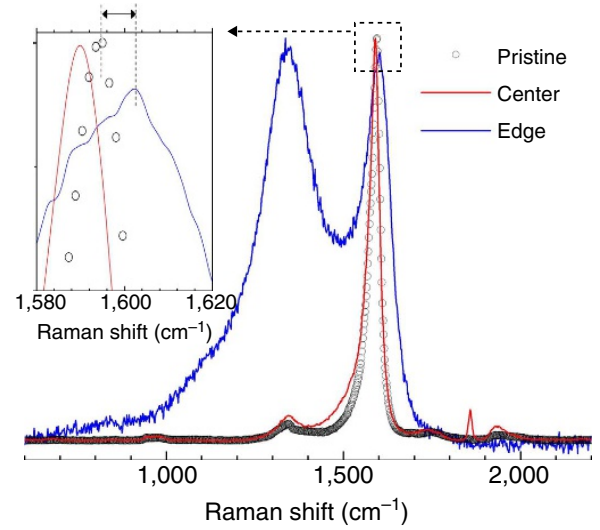

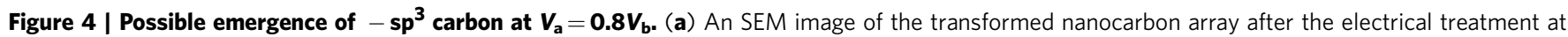
$0.8 V_{b}$ with 3,000 cycles. (d) High-magnification TEM images showing a lattice structure whose spacing is consistent with $-\mathrm{sp}^{2}$ carbon (see text), obtained from the centre-region of network. The inset figure of $\mathbf{b}$ shows the corresponding fast Fourier transform (FFT) pattern. (c) The carbon K-edge energy loss spectrum (EELS) obtained from the centre (red solid) and the edge (blue solid) of the sample as shown in $\mathbf{a}$. Signatures of $\mathrm{C}=\mathrm{C} \pi^{\star}$ bonds at the edge is reduced significantly, whereas a clear signature of $-\mathrm{sp}^{3}$ carbon appears in the spectrum (shown with an arrow, see text). The percentage of these $-\mathrm{sp}^{3}$ bonds could be obtained by comparing the Raman spectra of (d) the pristine samples (black circles), the central region of the voltage-pulse-cycled samples (red solid) and the edges of the same array (blue solid). The inset is the Raman spectra zoomed into the G-band range, which shows a red-shift in the central region consistent with the formation of multi-layered graphitic $\mathrm{sp}^{2} \mathrm{structures}$, and a blue shift of $12.4 \mathrm{~cm}^{-1}$ at the edges, which corresponds to the $\sim 17 \%$ of $\mathrm{sp}^{3}$ carbon structures (see text). Scale bars in (a), $200 \mathrm{~nm}$; in (b), $1 \mathrm{~nm}$.

However, a few relevant mechanisms are important to discuss. First, the instantaneous local temperature of the SWCNTs because of periodic voltage-pulse cycling could reach up to $1,200 \mathrm{~K}$ at $V_{\mathrm{a}}=0.8 V_{\mathrm{b}}$ (see Method section), and thermally induced changes in $-\mathrm{sp}^{2}$ carbon structures are usually negligible at such temperatures ${ }^{28}$. Moreover, the transformations are more effective under rapid voltage-switching (smaller values of $t_{\mathrm{on}}$ ) rather than higher heat-generating (larger values of $t_{\text {on }}$ ) cycles. This indicates that Joule heating alone is not sufficient to explain the transformation process. Second, the formation of bundles and the resulting densification of SWCNTs at the initial stages of cycling indicate that the applied voltage-pulses induce coherent mechanical forces that move nanotubes closer to each other. However, although these forces can move the nanotubes into intimate proximity, they alone are not likely to trigger the entire coalescence phenomenon. A third factor is the role of electromigration in triggering the transformations ${ }^{24,25,49}$. At high current densities, electrons can transfer significant momentum to atoms causing them to migrate from their parent sites, and such migration events rapidly grow in number at elevated temperatures (such as in our case). However, the effectiveness of electromigration alone in driving the entire sequence of transformations is difficult to quantify. We believe that a combination of all these above effects may be playing a role in bringing about the allotropic transformations. We speculate that the nanoscale forces that initially align the nanotubes bring them to van der Waals proximities. Thermal fluctuations and electromigration may cause these atoms to move out of their lattice sites, and these mobile atoms minimize their energies by forming linear chains between two SWNCTs (Fig. 2a) that forms a junction between nanotubes and give rise to the CIMs in Raman spectroscopy. With continued cycling, the junction grows wider and eventually becomes comparable in size with the nanotube diameters and the nanotubes release mechanical strain by coalescing into larger-diameter structures. Very likely, in-situ high-resolution TEM studies and atomistic simulations may be necessary for a better understanding of the overall transformation process steps.

In summary, we are able to transform SWCNT networks to other predetermined $\mathrm{sp}^{2}$ carbon nanostructures (SWCNTs with a larger diameter, MWNTs or MGNRs) by controlling the electrical parameters, such as alternating voltage-pulse magnitudes, sourceon-time and the number of switching cycles applied to the CNT networks. To our knowledge, this is the first demonstration of atomic-level engineering of molecular junctions and controlled structural transformations in CNT networks without increasing 
structural defects. The applied voltage, periodic polarity change and associated electrical force activate a coalescence-induced reconfiguration of $\mathrm{sp}^{2}$ hybridizion. The method can be facile and easily scalable, which will allow us to tailor the desired molecular structure and physical properties of nanocarbon networks for applications, such as electronic devices, energy storage systems and multifunctional membranes and fibres.

\section{Methods}

Fabrication of highly aligned SWCNTs. The basic steps for building organized SWCNT lateral architectures fabricated using the template-guided fluidic assembly process $^{30-32}$, are as follows. First, a $300-\mathrm{nm}$-thick polymethyl methacrylate photoresist is deposited on a substrate and baked at $160^{\circ} \mathrm{C}$ for $90 \mathrm{~s}$. Second, polymethyl methacrylate patterns are constructed on the substrate using electronbeam lithography to build nanoscale channels, which form templates for building the test architectures and these templates are then developed in the solution (methyl isobutyl ketone/isopropyl alcohol $=1: 3$ ) for $100 \mathrm{~s}$ followed by a 30-s rinse in isopropyl alcohol solution. Next, these template substrates are dip-coated in a $0.23 \mathrm{wt} \%$ high-purified SWCNT solution (Supplementary Table 1 and Supplementary Notes) at a constant pulling rate of $0.1 \mathrm{~mm} \mathrm{~min}^{-1}$. The dip-coating processes result in stable and densely aligned SWCNT lateral network architectures on the substrate having well-defined shapes at the nanoscale. Finally, the photoresist is removed to obtain well-organized and aligned SWCNT lateral networks. For the fabrication of contact electrodes consisting of $\operatorname{Ti}(5 \mathrm{~nm}) /$ $\mathrm{Au}(150 \mathrm{~nm})$, the same fabrication steps are applied except for a dip-coating process described above.

CNTs density in the network array. The networks made from dip-coating techniques typically have the smallest surface roughness and close-packing structures with $80 \%$ dense CNTs (based on Supplementary Fig. 1d,e). The cross-sectional area is approximately $18,000 \mathrm{~nm}^{2} \pm 3,000 \mathrm{~nm}^{2}$ (based on Supplementary Fig. $1 \mathrm{c}, \mathrm{f})$. Therefore, the number of CNTs in the network of $2 \mu \mathrm{m}^{2}$ dimension is calculated as about $(4 \pm 0.5) \times 10^{4}\left((2 \pm 0.25) \times 10^{12} \mathrm{~cm}^{-2}\right)$.

Transformation processes of SWCNT arrays. A Keithley 2,400 sourcemeter was used to apply voltage and to measure the resistance across the two terminal CNT devices. All the measurements were done under high vacuum $\left(P<10^{-5} \mathrm{Torr}\right)$ in a Janis Research ST-500 cryogenic probe station to reduce radial heat losses through gas convection and to avoid burning the devices. Voltage sweeps were applied through CNT arrays to find the breakdown voltage. Most of the CNT arrays failed at a maximum current density of about $4.3 \times 10^{7} \mathrm{~A} \mathrm{~cm}^{-2}$, and the breakdown voltage $\left(V_{\mathrm{b}}\right)$ was $2.7 \mathrm{~V}$ on the TEM window (Fig. 1b). By sweeping the voltage over a suitable range, we observe a critical voltage for which the graphitization process is speeded up. At the same time, applied voltages too close to $V_{\mathrm{b}}$ often leads to the breakdown of the nanotube (Supplementary Fig. 6). Therefore, $V_{\mathrm{b}}$ for these quasiparallel SWCNT networks was found to be an important characterization parameter. The $V_{\mathrm{b}}$ in our device could be predicted statistically by $V_{\mathrm{b}}=-2.14 \times$ $I_{1 V}+9.13$, here $I_{1 \mathrm{~V}}[\mathrm{~mA}]$ is a current at $1 \mathrm{~V}$. Arrays with a nearly similar current density were used for testing the reproducibility of the transformation process. For the structural engineering of the CNT arrays, repetitive positive and negative voltages of $0.4 V_{\mathrm{b}}, 0.6 \mathrm{~V}_{\mathrm{b}}$ and $0.8 \mathrm{~V}_{\mathrm{b}}$, which the electrical powers were in the range of $2-3,6-8$ and $10-15 \mathrm{~mW}$, respectively, under our device condition (for $\mathrm{SiO}_{2} / \mathrm{Si}$ substrate, see Supplementary Fig. 1g and Supplementary Methods), were each applied at $1 \mathrm{~Hz}$ for 3,000 cycles at $180^{\circ} \mathrm{C}(453 \mathrm{~K})$ between the two electrodes on the $\mathrm{CNT}$ arrays. Although the external temperature is too low to promote any $\mathrm{C}-\mathrm{C}$ bond rearrangement, this temperature showed a better effect than room temperature $(300 \mathrm{~K})$ for the engineering of the SWCNT arrays into new nanostructures. A higher external temperature caused wall defects and damage to the SWCNT arrays under each applied voltage. After transformation processes of SWCNT arrays, the devices were cooled slowly to prevent fracture due to the rapid cooling.

Temperature by electrical energy. Breakdown occurs when the maximum temperature of the tube reaches the value of the breakdown temperature, which allows the extraction of a simple expression for the breakdown voltage of SWCNTs, including heat generation from Joule self-heating and heat loss to the substrate ${ }^{50,51}$

$$
V_{\mathrm{BD}}=g L\left(T_{\mathrm{BD}}-T_{0}\right) / I_{\mathrm{BD}}+I_{\mathrm{BD}} R_{\mathrm{C}}
$$

Therefore, the maximum temperature at the breakdown voltage is explained as:

$$
T_{\mathrm{BD}}=\left(P_{\mathrm{BD}}-I_{\mathrm{BD}}^{2} R_{\mathrm{C}}\right) / g L+T_{0}
$$

Here, $T_{\mathrm{BD}}$ is the maximum temperature, $P_{\mathrm{BD}}$ is the breakdown power, the combined resistance of the source and drain contacts, $R_{\mathrm{C}}$ is estimated from the inverse slope of the low-bias $I_{\mathrm{D}}-V_{\mathrm{SD}}$ plot, $R_{\mathrm{C}} \approx\left(\mathrm{d} I_{\mathrm{D}} / \mathrm{d} V_{\mathrm{SD}}\right)^{-1}, g$ is the heat dissipation coefficient in the substrate per unit length and $L$ is length of CNT network. From the equation, the calculated maximum temperature is about $1,335 \mathrm{~K}$ $\left(1,062^{\circ} \mathrm{C}\right)$. At the $0.8 V_{\mathrm{b}}$, the temperature is calculated as about $1,200 \mathrm{~K}$.
SEM and TEM observation. The CNT arrays were prepared on specially designed chips with a window of $40 \times 40 \mu^{2}$ for SEM and TEM imaging. The CNT arrays were suspended on an electron transparent window by employing a two-probe device architecture. Two micro-heater electrodes of $10 \mu \mathrm{m}$ in width were employed around the arrays on the chip for the external heating. The TEM measurements were carried out in a JEOL-3011 high-resolution TEM instrument using an accelerating voltage of $300 \mathrm{kV}$. The allotropes cover a significant area of the devices, which was verified by taking TEM images at different positions over the sample.

Raman measurements. Raman spectroscopy (LabRAM HR 800, HORIBA Jobin Yvon, HORIBA) was used to investigate structure changes of CNT arrays as the applied voltage and the numbers of cycles were changed. The laser excitation wavelengths used were $532 \mathrm{~nm}(2.33 \mathrm{eV})$ and $633 \mathrm{~nm}(1.92 \mathrm{eV})$, the exposure time was $5 \mathrm{~s}$ per spectrum, and the number of data accumulations was 10. A 600 lines per $\mathrm{mm}$ grating was used, and the confocal hole diameter was set to $100 \mu \mathrm{m}$ (Supplementary Notes).

Electrical resistance measurements. To obtain a reasonable estimate of the contact resistance, four equally spaced identical electrodes were fabricated using a magnetron sputter-coater. The estimation used for the interfacial contact resistance assumes that the two-terminal resistance between any two electrodes is the sum of the device resistance of the CNT arrays and the interfacial contact resistance at each contact $^{52}$. From the two-terminal $I-V$ characteristics, the resistance $R$ was measured in all test structures, which have a lateral width of $1 \mu \mathrm{m}$. The four contact pads were separated by a distance of $2 \mu \mathrm{m}$ from each other. $I-V$ measurements were conducted between the pairs of contacts A-B, B-C, C-D, A-C and B-D. The contact resistance at contact pad $\mathrm{B}$ and $\mathrm{C}\left(R_{\mathrm{B}}^{c}\right.$ and $\left.R^{c} \mathrm{C}\right)$ can be written as

$$
\begin{aligned}
& R_{\mathrm{B}}^{c}=\frac{R_{\mathrm{AB}}+R_{\mathrm{BC}}-R_{\mathrm{AC}}}{2} \\
& R_{\mathrm{C}}^{c}=\frac{R_{\mathrm{BC}}+R_{\mathrm{CD}}-R_{\mathrm{BD}}}{2}
\end{aligned}
$$

where $R_{\mathrm{AB}}=R_{\mathrm{A}}^{c}+R_{\mathrm{AB}}^{d}+R_{\mathrm{B}}^{c}, R_{\mathrm{BC}}=R_{\mathrm{B}}^{c}+R_{\mathrm{BC}}^{d}+R_{\mathrm{C}}^{c}, R_{\mathrm{AC}}=R_{\mathrm{A}}^{c}+R_{\mathrm{AC}}^{d}+R_{\mathrm{C}}^{c}$, $R_{\mathrm{CD}}=R_{\mathrm{C}}^{c}+R_{\mathrm{CD}}^{d}+R_{\mathrm{D}}^{c}$ and $R_{\mathrm{BD}}=R_{\mathrm{B}}^{c}+R_{\mathrm{BD}}^{d}+R_{\mathrm{D}}^{c}$. The resistance $R_{\mathrm{BC}}^{d}$ is defined as a device resistance between a contact pad B and C (Supplementary Fig. 8a). Therefore, the two contact resistances and the resistances of the CNT array itself were all calculated from data taken on five pairs of device resistances and the values were compared before and after the CNT array treatment (Supplementary Fig. $8 \mathrm{~b}-\mathrm{d})$.

Thermal conductivity measurements. The measurement of thermal conductivity was performed by using a self-heating $3 \omega$ technique ${ }^{53,54}$. The $3 \omega$ signal correlates with the thermal conductivity through the following equation ${ }^{53,54}$,

$$
V_{3 \omega, \mathrm{rms}}=\frac{4 I^{3} R R^{\prime} L}{\pi^{4} k S}
$$

where $L, R$ and $S$ are the distance between the contacts, the electrical resistance and the cross-sectional area of the sample, respectively. $R^{\prime}=(\delta R / \delta T)$ is the temperature gradient of the resistance at the chosen temperature and $k$ is the thermal conductivity. The $3 \omega$ method was used by the four-point-probe third harmonic characterization method to eliminate the contact resistance and to avoid related spurious signals. The $3 \omega$ signals can be used for measuring the thermal conductivity of the SWCNT arrays. A lock-in amplifier (Stanford Research System SR850) was used for obtaining the $3 \omega$ signals by amplifying the small voltage and removing the noise. An AC current source (Keithley 6221) was used to provide a stable current supply. All the measurements including the resistance, temperature and $3 \omega$ signals were done under high vacuum $\left(P<10^{-5}\right.$ Torr $)$ in a Janis Research ST-500 cryogenic probe station to reduce radial heat losses through gas convection. The temperature coefficient of the resistance should be measured in order to obtain the thermal conductivity based on equation 5 . The resistance-temperature coefficient of the CNT arrays themselves was also measured over the small temperature range of $296-300 \mathrm{~K}$, which is close to the measurement temperature of the $3 \omega$ signal (see Supplementary Fig. 9). The resistance change of the pristine SWCNTs as a function of temperature was about $53.7 \Omega \mathrm{K}^{-1}$. Those of the samples treated at $0.4 V_{\mathrm{b}}$ and $0.6 V_{\mathrm{b}}$ showed a negative resistance temperature $(R(T))$ value of $-26.2 \Omega \mathrm{K}^{-1}$ and $-9.03 \Omega \mathrm{K}^{-1}$, respectively. On the other hand, $R(T)$ showed a positive value of $1.6 \Omega \mathrm{K}^{-1}$ for the sample treated at $0.8 \mathrm{~V}_{\mathrm{b}}$. The third-harmonic voltage measured at the frequency of $1,000 \mathrm{~Hz}$ is shown in Supplementary Fig. 9. By fitting these results according to equation (5), the exponent of the current amplitude $I_{0}$ was found to be $2.8-3.1$ for the samples. These values are very close to the third power predicted from theory. The calculated thermal conductivities were $58.5,63.5,13.1$ and $301.2 \mathrm{~W} \mathrm{mK}^{-1}$ for the pristine SWCNTs, and samples treated at $0.4 V_{\mathrm{b}}, 0.6 V_{\mathrm{b}}$ and $0.8 V_{\mathrm{b}}$, respectively (Fig. $3 \mathrm{~d}$ ). We assume that the factor of five increase of the effective thermal conductivity in the array treated at $0.8 V_{\mathrm{b}}$ is caused by the highly reduced junction scattering and the thermal pathways to $\mathrm{sp}^{3}$ structures. 


\section{References}

1. Volder, M., Tawfick, S., Baughman, R. H. \& Hart, A. J. Carbon nanotubes: present and future commercial applications. Science 339, 535-539 (2013).

2. Cao, Q. et al. Arrays of single-walled carbon nanotubes with full surface coverage for high-performance electronics. Nat. Nanotech. 8, 180-186 (2013).

3. Zhang, X. et al. Ultrastrong, stiff and lightweight carbon-nanotube fibers. Adv. Mater. 19, 4198-4201 (2007)

4. Lin, H. et al. Conducting polymer composite film incorporated with aligned carbon nanotubes for transparent, flexible and efficient supercapacitor. Sci. Rep. 3, 1353 (2013).

5. Srivastava, A. et al. Carbon nanotube filters. Nat. Mater. 3, 610-614 (2004).

6. Wu, Y. et al. Conformal $\mathrm{Fe}_{3} \mathrm{O}_{4}$ sheath on aligned carbon nanotube scaffolds as high-performance anodes for lithium ion batteries. Nano Lett. 13, 818-823 (2013)

7. Vilatela, J. J., Elliott, J. A. \& Windle, A. H. A model for the strength of yarn-like carbon nanotube fibers. ACS Nano 5, 1921-1927 (2011).

8. Hirsch, A. The era of carbon allotropes. Nat. Mater. 9, 868-871 (2010).

9. Jiao, L. et al. Narrow graphene nanoribbons from carbon nanotubes. Nature 458, 877-880 (2009).

10. Hu, M. et al. Compressed carbon nanotubes: a family of new multifunctional carbon allotropes. Sci. Rep. 3, 1331 (2013).

11. Krasheninnikov, A. V. \& Banhart, F. Engineering of nanostructured carbon materials with electron or ion beams. Nat. Mater. 6, 723-733 (2007).

12. Wei, D. \& Liu, Y. The intramolecular junctions of carbon nanotubes. Adv. Mater. 20, 2815-2841 (2008).

13. Endo, M. et al. Coalescence of double-walled carbon nanotubes: formation of novel carbon bicables. Nano Lett. 4, 1451-1454 (2004).

14. Terrones, M. et al. Molecular junctions by joining single-walled carbon nanotubes. Phys. Rev. Lett. 89, 0755051-0755054 (2002).

15. Yao, Z., Postma, H. W. C., Balents, L. \& Dekker, C. Carbon nanotube intramolecular junctions. Nature 402, 273-276 (1999).

16. Papadopoulos, C. et al. Electronic transport in Y-junction carbon nanotubes. Phys. Rev. Lett. 85, 3476-3479 (2000).

17. Zhou, C. W., Kong, J., Yenilmez, E. \& Dai, H. J. Modulated chemical doping of individual carbon nanotubes. Science 290, 1552-1555 (2000)

18. Bandaru, P. R., Daraio, C., Jin, S. \& Rao, A. M. Novel electrical switching behaviour and logic in carbon nanotube Y-junctions. Nat. Mater. 4, 663-666 (2005).

19. Kis, A. et al. Reinforcement of single-walled carbon nanotube bundles by intertube bridging. Nat. Mater. 3, 153-157 (2004)

20. Terrones, M. et al. Coalescence of single-walled carbon nanotubes. Science $\mathbf{2 8 8}$, $1226-1229$ (2000).

21. Li, J. \& Banhart, F. The engineering of hot carbon nanotubes with a focused electron beam. Nano Lett. 4, 1143-1146 (2004).

22. Yao, Y. et al. Temperature-mediated growth of single-walled carbon-nanotube intramolecular junctions. Nat. Mater. 6, 283-286 (2007).

23. Collins, P. G., Arnold, M. S. \& Avouris, P. Engineering carbon nanotubes and nanotube circuits using electrical breakdown. Science 292, 706-709 (2001).

24. Jia, X. et al. Controlled formation of sharp zigzag and armchair edges in graphitic nanoribbons. Science 323, 1701-1705 (2009).

25. Jin, C., Suenaga, K. \& Iijima, S. Plumbing carbon nanotubes. Nat. Nanotech. 3, 17-21 (2008).

26. Yuzvinsky, T. D. et al. Shrinking a carbon nanotube. Nano Lett. 6, 2718-2722 (2006)

27. Mølhave, K. et al. Transmission electron microscopy study of individual carbon nanotube breakdown caused by joule heating in air. Nano Lett. 6, 1663-1668 (2006).

28. Gutiérrez, H. R., Kim, U. J., Kim, J. P. \& Eklund, P. C. Thermal conversion of bundled carbon nanotubes into graphitic ribbons. Nano Lett. 5, 2195-2201 (2005).

29. Romo-Herrera, J. M. et al. Covalent 2D and 3D networks from 1D nanostructures: Designing new materials. Nano Lett. 7, 570-576 (2007).

30. Jaber-Ansari, L. et al. Mechanism of very large scale assembly of SWNTs in template guided fluidic assembly process. J. Am. Chem. Soc. 131, 804-808 (2009).

31. Xiong, X. et al. Building highly organized single-walled carbon nanotube networks using template guided fluidic assembly. Small 3, 2006-2010 (2007)

32. Somu, S. et al. Topological transition in carbon nanotube networks via nanoscale confinement. ACS Nano 4, 4142-4148 (2010).

33. Yao, Z., Kane, C. L. \& Dekker, C. High-field electrical transport in single-wall carbon nanotubes. Phys. Rev. Lett. 84, 2941-2944 (2004).

34. Yoon, M. et al. Zipper mechanism of nanotube fusion: theory and experiment. Phys. Rev. Lett. 92, 0755041-0755044 (2004).

35. Ajayan, P. M., Ravikumar, V. \& Charlier, J.-C. Surface reconstructions and dimensional changes in single-walled carbon nanotubes. Phys. Rev. Lett. 81, 1437-1440 (1998).

36. Hernandez, E. et al. Fullerene coalescence in nanopeapods: a path to novel tubular carbon. Nano Lett. 3, 1037-1042 (2003).
37. Endo, M. et al. Atomic nanotube welders: boron interstitials triggering connections in double-walled carbon nanotubes. Nano Lett. 5, 1099-1105 (2005).

38. Endo, M. et al. Nanotube coalescence-inducing mode: a novel vibrational mode in carbon systems. Small 2, 1031-1036 (2006).

39. Fantini, C. et al. Resonance Raman study of linear carbon chains formed by the heat treatment of double-wall carbon nanotubes. Phys. Rev. B 73, 1934081-1934084 (2006)

40. Villalpando-Paez, F. et al. Raman spectroscopy study of heat-treated and boron-doped double wall carbon nanotubes. Phys. Rev. B 80, 0354191-03541914 (2009).

41. Filho, S. et al. Competing spring constant versus double resonance effects on the properties of dispersive modes in isolated single-wall carbon nanotubes. Phys. Rev. B 67, 035427-035434 (2003).

42. Papworth, A.P. et al. Electron-energy-loss spectroscopy characterization of the sp2 bonding fraction within carbon thin films. Phys. Rev. B 62, 12628-12631 (2000).

43. Okada, K., Kimoto, K., Komatsu, S. \& Matsumoto, S. Sp2 bonding distributions in nanocrystalline diamond particles by electron energy loss spectroscopy. J. Appl. Phys. 93, 3120-3122 (2003).

44. Castrucci, P. et al. Probing the electronic structure of carbon nanotubes by nanoscale spectroscopy. Nanoscale 2, 1611-1625 (2010).

45. Prawer, S. et al. The Raman spectrum of nanocrystalline diamond. Chem. Phys. Lett. 332, 93-97 (2000).

46. Mykhaylyka, O. O., Solonin, Y. M., Batchelder, D. N. \& Brydson., R. Transformation of nanodiamond into carbon onion. J. Appl. Phys. 97, 074302-074315 (2005).

47. Arenal, R. et al. Diamond nanowires and the insulator-metal transition in ultrananocrystalline diamond films. Phys. Rev. B 75, 195431-11 (2007)

48. Singha, A., Ghosh, A., Roy, A. \& Ray, N. R. Quantitative analysis of hydrogenated diamondlike carbon films by visible Raman spectroscopy. J. Appl. Phys. 100, 044910 (2006).

49. Sorbello, R. Theory of electromigration. Solid State Phys. 51, 159-231 (1997).

50. Pop, E., Mann, E. A., Goodson, K. E. \& Dai, H. Electrical and thermal transport in metallic single-wall carbon nanotubes on insulating substrates. J. Appl. Phys. 101, 093710 (2007)

51. Liao, A. et al. Thermal dissipation and variability in electrical breakdown of carbon nanotube devices. Phys. Rev. B 82, 205406 (2010).

52. Kim, Y. L. et al. Highly aligned scalable platinum-decorated single-wall carbon nanotube arrays for nanoscale electrical interconnects. ACS Nano 3, 2818-2826 (2009).

53. Li, B. et al. Ultra-thin SWNTs films with tunable, anisotropic transport properties. Adv. Funct. Mater. 21, 1810-1815 (2011).

54. Choi, T., Poulikakos, D., Tharian, J. \& Sennhauser, U. Measurement of the thermal conductivity of individual carbon nanotubes by the four-point three- $\omega$ method. Nano Lett. 6, 1589-1593 (2006).

\section{Acknowledgements}

Y.J.J. and H.Y.J. acknowledge the financial support from Fundamental R\&D Program for Core Technology of Materials in the Ministry of Knowledge Economy (MKE), Republic of Korea and National Science Foundation-CMMI grant (0927088). C.W.A. acknowledges the financial support from Global Frontier (CASE-2011-0032147), Basic Research (2013R1A1A2006973), and Nanomaterials (2014M3A7B6034494), MEST, Republic of Korea. S.K. acknowledges partial support from an NSF grant (ECCS-1202376). P.T.A. and M.S.D. acknowledge the EFRC-S3TEC Center supported by the DOE contract DE-SC0001299/DE-FG02-09ER46577.

\section{Author contributions}

H.Y.J., S.K., M.S.D. and Y.J.J. designed the experiments. H.Y.J., Y.L.K. and S.H. fabricated the samples; H.Y.J., P.T.A. and S.M.J. performed the Raman spectroscopy; H.Y.J., Y.L.K., J.K. and S.M.J. performed the SEM; H.Y.J., X.J. and C.W.A. performed the TEM; C.W.A. fabricated the special TEM chips; H.Y.J. and S.H. drew the schematics and H.Y.J. performed the electrical and thermal measurements. H.Y.J., P.T.A., S.K., M.S.D. and Y.J.J. wrote the manuscript.

\section{Additional information}

Supplementary Information accompanies this paper at http://www.nature.com/ naturecommunications

Competing financial interests: H.Y.J., P.T.A., C.W.A., M.S.D., S.K. and Y.J.J. are co-inventors on an unpublished patent application owned by Northeastern University (US Provisional Pat. App. Ser. No. 61/832,347). Authors not involved in the patent have no competing financial interests.

Reprints and permission information is available online at http://npg.nature.com/ reprintsandpermissions/

How to cite this article: Jung, H. Y. et al. Sculpting carbon bonds for allotropic transformation through solid-state re-engineering of $-\mathrm{sp}^{2}$ carbon. Nat. Commun. 5:4941 doi: $10.1038 /$ ncomms5941 (2014). 